\title{
Análise da identificação botânica em inventários florestais de planos de manejo sustentáveis no oeste paraense
}

O inventário florestal é uma importante ferramenta para o planejamento do manejo florestal sustentável, auxiliando na tomada de decisão quantos aos produtos florestais madeireiros e não madeireiros. Uma das informações mais importantes de um inventário florestal é a identificação botânica ou taxonômica, que usualmente é realizada por parabotânicos/parataxonomistas (mateiros). Estes profissionais normalmente não dispõem de metodologias científicas que garantam a correta identificação taxonômica das espécies, utilizando características morfológicas e nomes vernaculares, que por vezes são bastante variáveis de acordo com a região. O objetivo desta pesquisa é analisar a identificação botânica e taxonômica de inventários florestais de empreendimentos do oeste Paraense que solicitaram licenciamento para Plano de Manejo Florestal Sustentável (PMFS), com foco especial nas famílias botânicas de menor representatividade (com 01 ou 02 espécies). Como resultado foi verificado uma variedade de problemas e erros relacionados aos nomes científicos e vernaculares apresentados nos projetos, entre os principais: falta do autor, erro na grafia do gênero, erro na grafia do epíteto específico, erro na grafia do autor, nomenclatura desatualizada e uso de sinônimos e/ou nomes não aceitos. Foram encontradas também espécies que não ocorrem na região norte, e outras que nem existe registro de ocorrência no Brasil, assim como táxons que não são árvores, mas constavam nos inventários. Sugerimos uma maior rigorosidade na análise destes dados pelos órgãos licenciadores e principalmente um maior compromisso das empresas de consultoria com a coleta e identificação taxonômica das espécies.

Palavras-chave: Política Ambiental; Recursos Naturais; Floresta Amazônica.

\section{Analysis of botanical identification in forest inventories of sustainable management plans on wester Pará state, Brazil}

The forest inventory is an important tool for the planning sustainable forest management, assisting in the decision-making in relation to forest, such as timber and non-timber products. One of the most important information of a forest inventory is the botanical or taxonomic identification, which is usually performed by parabotanists/parataxonomists. These professionals normally do not have scientific methodologies that guarantee the correct taxonomic identification of the species, using morphological characteristics and vernacular names, which are sometimes quite volatile according to the region. The objective of this paper is to analyze the botanical and taxonomic identification of forest inventories of an enterprise that requested licensing for the Sustainable Forest Management Plan (SFMP), on western Pará state, Brazil, with a special focus on the least representative botanical families (01 or 02 species). As a result, a variety of problems and errors related to the scientific and vernacular names presented in the projects were verified, among the main ones: lack of the author, orthographic error of the genre and specific epithet, author orthographic error, outdated nomenclature and use of synonyms and/or names not accepted. As well as species that do not occur in the northern region, and others that do not even exist in Brazil and taxa that are not trees, but were included in the inventory. We suggest a greater stringent in the analysis of this data by the licensing agencies and mainly a greater commitment of the consulting companies with the collection and taxonomic identification of the species.

Keywords: Environmental Policy; Natural Resource; Amazonian Rainforests.

Topic: Planejamento, Gestão e Políticas Públicas Ambientais

Reviewed anonymously in the process of blind peer
Received: 09/05/2020

Approved: 03/07/2020

Rafael Lima Araújo Ferreira

Universidade Federal do Pará, Brasil

http://lattes.cnpq.br/4836515061363797

http://orcid.org/0000-0001-5400-2827

rafaelferreirax@gmail.com

Roberta Macedo Cerqueira

Universidade Federal do Pará, Brasil

http://lattes.cnpq.br/2863595777814509

rmcerqueira@ufpa.br

Rivaldo Costa Cardoso Junio

Universidade Federal Rural da Amazônia, Brasil

http://lattes.cnpq.br/2169420783752907

rivasforestry@gmail.com

6

DOI: 10.6008/CBPC2318-2881.2020.003.0014
Referencing this:

FERREIRA, R. L. A.; CERQUEIRA, R. M.; CARDOSO JUNIOR, R. C.. Análise da identificação botânica em inventários florestais de planos de manejo sustentáveis no oeste paraense. Nature and Conservation, v.13, n.3, p.136-145, 2020. DOI: http://doi.org/10.6008/CBPC23182881.2020.003.0014 


\section{INTRODUÇÃO}

O manejo da floresta sustentável constitui uma alternativa racional para a produção econômica. Através de um processo de gerenciamento permanente de áreas florestais e se utilizando de princípios, técnicas e normas para ordenar a produção, o manejo maximiza a produtividade e a eficiência (SILVA et al., 1985; QUEIROZ, 2012; SOUZA et al., 2013).

As áreas florestais submetidas ao manejo apresentam muitos benefícios: produtos madeireiros, múltiplos produtos e subprodutos não madeireiros, bens e serviços de regulação ambiental. Além de produção de informações que auxiliam nos estudos e desenvolvimento de políticas ambientais, por isso, administrar os recursos da floresta passa necessariamente por um bom planejamento, onde a coleta de dados, análise e interpretação dos diversos parâmetros sociais, ecológicos e econômicos, irá garantir um fluxo continuo de produtos e de serviços florestais, além da redução do risco de alterações ecológicas significativas (SILVA et al., 1985; QUEIROZ, 2012; SOUZA et al., 2013).

Segundo o Código Florestal Brasileiro:

O manejo sustentável é a administração da vegetação natural para a obtenção de benefícios econômicos, sociais e ambientais, respeitando-se os mecanismos de sustentação do ecossistema objeto do manejo e considerando-se, cumulativa ou alternativamente, a utilização de múltiplas espécies madeireiras ou não, de múltiplos produtos e subprodutos da flora, bem como a utilização de outros bens e serviços. (BRASIL, 2012).

O inventário florestal é o passo inicial e fundamental para o planejamento da propriedade florestal, dando suporte técnico-científico necessário para alcançar os objetivos do manejo, relacionando-o aos componentes ecológicos, florísticos, qualitativos e outros (SILVA et al., 1985; QUEIROZ, 2012; SOUZA et al., 2013). Outro aspecto importante, segundo Araújo (2006) é a possibilidade de projeções de ordem econômicas, referente à comercialização, tais como: cálculos de despesas e receitas esperadas, mercados a atingir, etc. O Inventário florestal visa avaliar as variáveis qualitativas e quantitativas da floresta e suas interrelações, assim como dinâmicas de crescimento e sucessão florestal, servindo de base para a formulação de planos de utilização dos produtos florestais, manejo e proposta de planos de desenvolvimento e política florestal de caráter regional ou nacional (QUEIROZ, 2012; ARAÚJO, 2006; VIBRANS et al., 2012).

Um dos componentes de grande importância do inventário florestal é a identificação botânica, realizada através do estudo taxonômico do material botânico coletado, que é preparado e depositado em herbários (MARTINS-DA-SILVA, 2002). Usualmente, conta com o conhecimento empírico de nativos conhecedores da área (mateiros/parabotânicos/parataxonomistas), que são profissionais que desenvolveram métodos de reconhecimento de espécies por meio de correlação de características morfológicas que mais se destacam, com nomes que os façam recordar daquela espécie. Desta forma, adotam predominantemente nomes vernaculares (comuns/populares) na determinação das espécies, e sem procedimentos de coleta botânica para conferência (PROCÓPIO et al., 2008).

O nome vernacular nos inventários florestais traz alguns problemas como: a imprecisão de ocorrência geográfica; a criação de blocos de espécies para um único nome comum; comprometimento dos cálculos dos índices de manutenção (selecionadas para corte); problemas na comercialização; prejuízos econômicos; 
exploração de forma desordenada e não sustentável; redução da diversidade e ainda espécies raras e sem importância econômica que por vezes não possuem denominações e acabam sendo relegadas (MARTINSDA-SILVA, 2002; JUDD et al., 2009)

Algumas empresas e/ou profissionais comumente lançam mão de listas de correspondência entre nomes vernaculares e nomes científicos, geralmente disponíveis nos órgãos licenciadores, como é o caso da Secretaria de Estado de Meio Ambiente e Sustentabilidade do Pará (SEMAS). Por vezes, esta correspondência de nomes é feita sem seguir critérios científicos, morfológicos ou ecológicos (BOTOSSO, 2009). Esta metodologia pode gerar imprecisão na identificação das espécies do inventário, além de muitas vezes, menosprezar as espécies consideradas comercialmente não valiosas.

O presente trabalho teve como objetivo verificar a identificação taxonômica de espécies botânicas citadas em inventários florestais realizados para a elaboração de Plano de Manejo Florestal Sustentável (PMFS) do oeste Paraense, assim como levantar os principais erros relacionados a estas identificações, colaborando assim com a preservação da flora local e regional.

\section{MATERIAIS E MÉTODOS}

Foram selecionados 60 processos de Planos de Manejo Florestal Sustentáveis dos municípios do oeste Paraense (Figura 01), contendo informações de Inventário Florestal a 100\% (IF100\%) e demais informações dos empreendimentos e das análises dos setores da Secretaria de Estado de Meio Ambiente e Sustentabilidade do Pará (SEMAS). Todos os processos foram protocolados na SEMAS no período de 2017 a junho de 2019, período em que o órgão digitalizou todo o conteúdo dos processos em seu sistema interno, o que facilitou a coleta das informações.

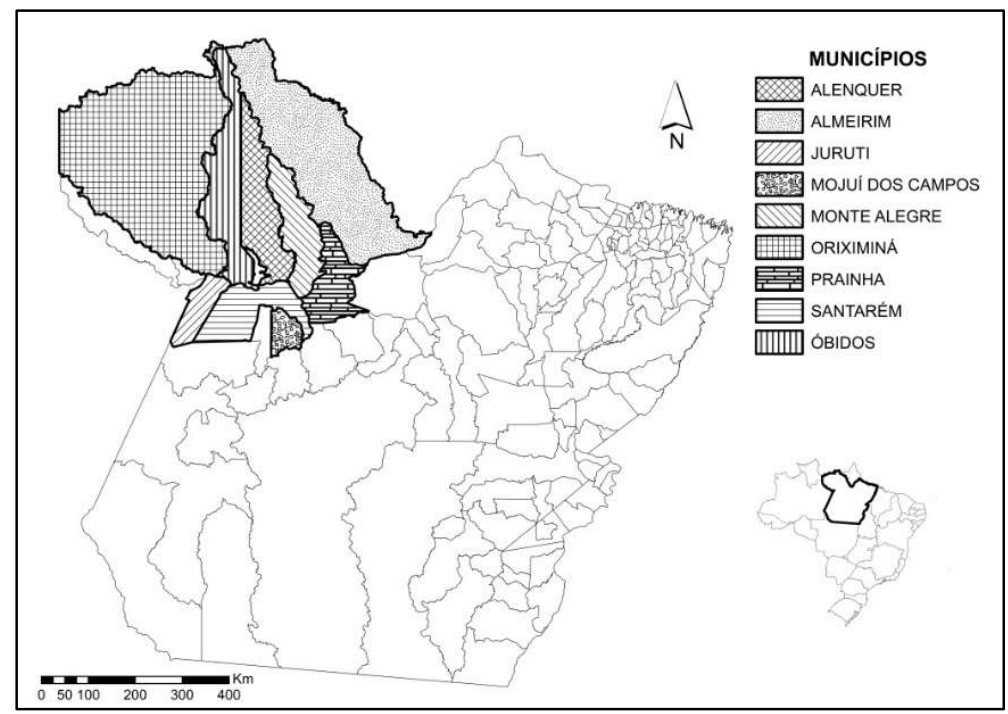

Figura 1: Mapa de localização dos Municípios alvo das análises.

Numa planilha do programa Excel ${ }^{\circledast}$, construiu-se um banco de dados com as seguintes informações: Município, Número do Processo, Nome Científico, Nome Popular, Gênero e Famílias Botânicas. Estes dados foram verificados, de forma a permanecer apenas registro de espécies contendo o nome científico (gênero 
e epíteto específico). Após esta etapa inicial, foram utilizados os sites da REFLORA - Flora do Brasil 2020ㄹ, Trópicos - Missouri Botanical Garden², UTAD - Jardim Botânico da Universidade de Trás-os-Montes e Alto Douro $^{3}$ e a Splink - Rede SpeciesLink ${ }^{4}$, que são plataformas de consulta botânica. Estes sites foram utilizados para verificar a identificação dos gêneros e das famílias botânica, de forma que estas informações foram posteriormente acrescidas ao banco de dados.

Para aprofundamento das análises, selecionaram-se as famílias botânicas com a menor representatividade de espécies (máximo duas), para verificar a identificação da família, gênero e espécie, ocorrência geográfica e forma de vida, utilizando-se das ferramentas já mencionadas acima. Pressupõem que espécies de menor representatividade são aquelas que recebem menor atenção no momento da identificação e/ou possuem menor importância econômica. Priorizou-se para a identificação taxonômica o site Trópicos e para a verificação de ocorrência geográfica e forma de vida o site REFLORA.

\section{RESULTADOS}

\section{Resultado inicial da construção do banco de dados}

Como resultado inicial da construção do Banco de Dados, verificou-se que cinco (5) processos dos 60 analisados não tinham em seus IF100\% a presença dos nomes científicos (apenas com a nomenclatura vernacular); arquivos não estavam disponíveis (corrompidos); ou outros motivos que impediram a inclusão nas análises deste trabalho. Desta forma cinco (05) processos foram excluídos das análises, restando 55. Destes 55 processos e da análise de seus IF100\%, obtivemos como resultado a presença de 55 famílias botânicas e 590 espécies, conforme a Tabela 1:

Tabela 1: Lista do número (N) das famílias, espécies (sp.) e a proporção representativa do total de espécies em porcentagem (\%).

\begin{tabular}{llll}
\hline $\mathbf{N}$ & FAMílIA & sp. & \% \\
\hline 1 & Fabaceae Lindl. & 164 & 27,8 \\
2 & Moraceae Gaudich & 38 & 6,44 \\
3 & Sapotaceae Juss. & 34 & 5,76 \\
4 & Lauraceae Juss. & 32 & 5,42 \\
5 & Lecythidaceae A. Rich. & 28 & 4,75 \\
6 & Malvaceae Juss. & 27 & 4,58 \\
7 & Vochysiaceae A. St.-Hil. & 23 & 3,9 \\
8 & Apocynaceae Juss. & 20 & 3,39 \\
9 & Myristicaceae R. Br. & 18 & 3,05 \\
10 & Euphorbiaceae Juss. & 17 & 2,88 \\
11 & Anacardiaceae R. Br. & 15 & 2,54 \\
12 & Chrysobalanaceae R. Br. & 15 & 2,54 \\
13 & Burseraceae Kunth & 14 & 2,37 \\
14 & Rubiaceae Juss. & 12 & 2,03 \\
15 & Humiriaceae A. Juss. & 11 & 1,86 \\
16 & Annonaceae Juss. & 8 & 1,36 \\
17 & Bignoniaceae Juss. & 7 & 1,19 \\
18 & Calophyllaceae J. Agardh & 7 & 1,19 \\
19 & Combretaceae R. Br. & 7 & 1,19 \\
20 & Boraginaceae Juss. & 6 & 1,02 \\
21 & Meliaceae Juss. & 6 & 1,02 \\
& & &
\end{tabular}

\begin{tabular}{llll}
\hline $\mathbf{N}$ & FAMílIA & sp. & $\%$ \\
\hline 29 & Arecaceae Bercht. \& J. Presl & 3 & 0,51 \\
30 & Clusiaceae Lindl. & 3 & 0,51 \\
31 & Olacaceae R. Br. & 3 & 0,51 \\
32 & Phyllanthaceae Martinov & 3 & 0,51 \\
33 & Sapindaceae Juss. & 3 & 0,51 \\
34 & Elaeocarpaceae Juss. & 2 & 0,34 \\
35 & Fagaceae Dumort. & 2 & 0,34 \\
36 & Hypericaceae Juss. & 2 & 0,34 \\
37 & Melastomataceae Juss. & 2 & 0,34 \\
38 & Metteniusaceae H. Karst. ex Schnizl. & 2 & 0,34 \\
39 & Peraceae Klotzsch & 2 & 0,34 \\
40 & Polygonaceae Juss. & 2 & 0,34 \\
41 & Rhamnaceae Juss. & 2 & 0,34 \\
42 & Salicaceae Mirb. & 2 & 0,34 \\
43 & Simaroubaceae DC. & 2 & 0,34 \\
44 & Styracaceae DC. \& Spreng. & 2 & 0,34 \\
45 & Verbenaceae J. St.-Hil. & 2 & 0,34 \\
46 & Caryophyllaceae Juss. & 1 & 0,17 \\
47 & Cucurbitaceae Juss. & 1 & 0,17 \\
48 & Dilleniaceae Salisb. & 1 & 0,17 \\
49 & Goupiaceae Miers & 1 & 0,17
\end{tabular}

\footnotetext{
${ }^{1}$ reflora.jbrj.gov.br

${ }^{2}$ www.tropicos.org

3 jb.utad.pt/jardim

${ }^{4}$ http://splink.org.br/
} 


\begin{tabular}{llll}
22 & Myrtaceae Juss. & 6 & 1,02 \\
23 & Urticaceae Juss. & 6 & 1,02 \\
24 & Araliaceae Juss. & 4 & 0,68 \\
25 & Caryocaraceae Voigt & 4 & 0,68 \\
26 & Nyctaginaceae Juss. & 4 & 0,68 \\
27 & Proteaceae Juss. & 4 & 0,68 \\
28 & Rutaceae Juss. & 4 & 0,68 \\
\hline
\end{tabular}

\begin{tabular}{llll}
50 & Lythraceae J. St.-Hil. & 1 & 0,17 \\
51 & Malpighiaceae Juss. & 1 & 0,17 \\
52 & Opiliaceae Valeton & 1 & 0,17 \\
53 & Quiinaceae Choisy ex Engl. & 1 & 0,17 \\
54 & Rosaceae Juss. & 1 & 0,17 \\
55 & Solanaceae Juss. & 1 & 0,17 \\
\hline
\end{tabular}

\section{As inconsistências na nomenclatura}

Em um processo localizado no Município de Juruti, observou-se um gênero que não era encontrado em nenhum dos bancos de dados consultados, mas ao utilizar o buscador do Google, chegou-se ao inusitado resultado de que o nome Pyrrhura frontalis (Vieil.), pertence a uma espécie de pássaro, popularmente conhecido como 'Tiriba', sendo este, o mesmo nome vulgar utilizado no IF100\%. No mesmo processo, encontrou-se outra inconsistência na utilização do nome científico, desta vez quanto a ocorrência da espécie Sorbus domestica L., que no caso, tem sua ocorrência geográfica na Europa e África.

Em outro processo, outra 'espécie' verificada foi a Zanthoxylum phoilim Lam., cujo o epíteto específico não consta em nenhum dos bancos de dados pesquisados, apesar da existência do gênero. Outra situação observada foi a 'espécie' Parancarnia amacos, cujo gênero poderia ter problema de grafia, conforme a grafia próxima do gênero Parahancornia, mas assim como o Zanthoxylum, não existe registro da relação do gênero Parahancornia com o epíteto específico 'amacos'.

Encontramos também a presença do gênero seguido do autor, de forma que o nome parecia estar no formato binomial, conforme previsto nas regras de nomenclatura botânica, como foi o caso do Chaunochiton Benth. Durante a análise da 'espécie' Psidium dentale e Pouteria pachycarpa Pires, comumente chamadas de 'casca seca' e 'goiabão', verificou-se que estas espécies não constam em nenhum dos sistemas consultados.

\section{As famílias botânicas com menor representatividade}

Dentre as 55 famílias botânicas disponíveis no banco de dados, foram selecionadas 22, considerando a existência de no máximo duas espécies por família, conforme as famílias em destaque na Tabela 2. Dentre essas, verificou-se a que a Goupiaceae apresentou mais de $96 \%$ de presença nos inventários analisados, contendo apenas uma espécie (Goupia glabra Aubl.).

Tabela 2: As Famílias selecionadas e com maior frequência nos IF100\% analisados.

\begin{tabular}{llll}
\hline FAMílIA & OCORRÊNCIA NOS IF & $\%$ & № ESPÉCIES \\
\hline Goupiaceae & 53 & 96,36 & 1 \\
Simaroubaceae & 42 & 76,36 & 2 \\
Salicaceae & 34 & 61,82 & 2 \\
Melastomataceae & 14 & 25,45 & 2 \\
\hline
\end{tabular}

As espécies das famílias botânicas com menor representatividade

O resultado obtido com o uso das ferramentas de consulta para cada nome científico está demonstrado na Tabela 3, com a coluna nome corrigido, onde foi inserido o nome correto para o táxon apresentado no IF100\%, priorizando o nome disponível no Trópicos, site internacional de atualização mais 
recente.

Tabela 3: Famílias e seus representantes, alguns tiveram seus nomes corrigidos (estão com *).

\begin{tabular}{|c|c|c|c|}
\hline FAMÍLIA & GÊNERO & NOME USADO NO IF100\% & NOME CORRIGIDO \\
\hline $\begin{array}{l}\text { Caryophyllacea } \\
\text { e }\end{array}$ & Drymaria & Drymaria cordate* & Drymaria cordata (L.) Willd. ex Schult. \\
\hline Cucurbitaceae & Cayaponia & Cayaponia tubulosa Cogn. & Cayaponia tubulosa Cogn. \\
\hline Dilleniaceae & Curatella & Curatella americana $\mathrm{L}$. & Curatella americana $\mathrm{L}$. \\
\hline Elaeocarpaceae & Sloanea & Sloanea brachysepala Ducke* & Sloanea laurifolia (Benth.) Benth. \\
\hline Elaeocarpaceae & Sloanea & Sloanea nitida G.Don & Sloanea nitida G.Don \\
\hline Fagaceae & Castanea & Castanea sativa* & Castanea sativa Mill. \\
\hline Fagaceae & Catostema & Catostema albuquerquei Paula* & Catostemma albuquerquei Paula \\
\hline Goupiaceae & Goupia & $\begin{array}{l}\text { Goupia glabra, Goupia glabra Aubl., Goupia glabra } \\
\text { Aublet* }\end{array}$ & Goupia glabra Aubl. \\
\hline Hypericaceae & Vismia & Vismia brasiliensis Choisy & Vismia brasiliensis Choisy \\
\hline Hypericaceae & Vismia & Vismia guianensis Pers. ${ }^{*}$ & Vismia guianensis (Aubl.) Choisy \\
\hline Lythraceae & Lafoensia & Lafoensia glyptocarpa Koehne & Lafoensia glyptocarpa Koehne \\
\hline Malpighiaceae & Byrsonima & Byrsonima aerugo Sagot & Byrsonima aerugo Sagot \\
\hline $\begin{array}{l}\text { Melastomatace } \\
\text { ae }\end{array}$ & Mouriri & Mouriri callocarpa Ducke, Mouriria calocarpa* & Mouriri collocarpa Ducke \\
\hline $\begin{array}{l}\text { Melastomatace } \\
\text { ae }\end{array}$ & Mouriri & Mouriri guyanensis Aubl.* & Mouriri guianensis Aubl. \\
\hline $\begin{array}{l}\text { Metteniusacea } \\
\text { e }\end{array}$ & Emmotum & Emmotum fagifolium Desv.* & Emmotum fagifolium Desv. ex Ham. \\
\hline $\begin{array}{l}\text { Metteniusacea } \\
\mathrm{e}\end{array}$ & Emmotum & Emmotum nitens (Benth.) Miers & Emmotum nitens (Benth.) Miers \\
\hline Opiliaceae & Agonandra & Agonandra brasiliensis Miers* & $\begin{array}{l}\text { Agonandra brasiliensis Miers ex Benth. \& } \\
\text { Hook.f. }\end{array}$ \\
\hline Peraceae & Pera & Pera glabrata (Schott) Baill.* & Pera glabrata (Schott) Poepp. ex Baill. \\
\hline Peraceae & $\begin{array}{l}\text { Pogonopho } \\
\text { ra }\end{array}$ & Pogonophora schomburgkiana Miers ex Benth* & \begin{tabular}{|l} 
Pogonophora schomburgkiana Miers ex \\
Benth.
\end{tabular} \\
\hline Polygonaceae & Coccoloba & Coccoloba latifolia Lam. & Coccoloba latifolia Lam. \\
\hline Polygonaceae & Triplaris & Triplaris surinamensis Cham.* & Triplaris weigeltiana (Rchb.) Kuntze \\
\hline Quiinaceae & Lacunaria & Lacunaria jenmani (Oliv.) Ducke* & Lacunaria jenmanii (Oliv.) Ducke \\
\hline Rhamnaceae & Ziziphus & Ziziphus cinnamomum Triana \& Planch* & Ziziphus cinnamomum Triana \& Planch. \\
\hline Rhamnaceae & Zizyphus & Zizyphus platyphylla Reissek* & Ziziphus platyphylla Reissek \\
\hline Rosaceae & Sorbus & Sorbus domestica L. & Sorbus domestica L. \\
\hline Salicaceae & Casearia & Casearia sylvestris* & Casearia sylvestris Sw. \\
\hline Salicaceae & Laetia & Laetia procera, Laetia procera (Poepp.) Eichler* & Laetia procera (Poepp.) Eichler \\
\hline Simaroubaceae & Simaba & Simaba guianensis* & Simaba guianensis Aubl. \\
\hline Simaroubaceae & Simarouba & Simarouba amara, Simarouba amara Aubl.* & Simarouba amara Aubl. \\
\hline Solanaceae & Acnistus & Acnistus arborescens Schltr.* & Acnistus arborescens (L.) Schltdl. \\
\hline Styracaceae & Styrax & Styrax acuminatum, Styrax acuminatum Pohl* & Styrax acuminatus Pohl \\
\hline Styracaceae & Styrax & Styrax ferrugineum* & Styrax ferrugineus Nees \& Mart. \\
\hline Verbenaceae & \begin{tabular}{|l} 
Citharexylu \\
$m$
\end{tabular} & Citharexylum cinereum L. & Citharexylum cinereum $\mathrm{L}$. \\
\hline Verbenaceae & $\begin{array}{l}\text { Citharexylu } \\
m\end{array}$ & Citharexylum myrianthum Cham. & Citharexylum myrianthum Cham. \\
\hline
\end{tabular}

Como resultado da consulta da ocorrência geográfica das espécies listadas acima, observou-se que 25 espécies ocorrem na Região Norte do Brasil, ou seja, nove (09) das 34 espécies não tem registro de ocorrência na Região Norte, região dos processos analisados. Dentre estas nove (09) espécies, três (03) não tem registro de ocorrência no Brasil. Como resultado das formas de vida, verificou-se a existência de duas (02) espécies não arbóreas: Drymaria cordata (L.) Willd. ex Schult. que é classificada como erva, e Cayaponia tubulosa Cogn., classificada como liana/trepadeira. 
Tabela 4: Espécies e o registro de ocorrência, fora da área de estudo.

\begin{tabular}{|l|l|}
\hline NOME CORRIGIDO & OCORRÊNCIA \\
\hline Castanea sativa Mill. & Europa e Ásia \\
\hline Sorbus domestica L. & Europa e África \\
\hline Citharexylum cinereum L. & América central \\
\hline Vismia brasiliensis Choisy & Sudeste (Minas Gerais, São Paulo) \\
\hline Lafoensia glyptocarpa Koehne & Nordeste (Bahia, Pernambuco) \\
\hline Ziziphus platyphylla Reissek & $\begin{array}{l}\text { Nordeste (Alagoas, Bahia, Ceará, Paraíba, Pernambuco, Rio Grande do Norte) e Sudeste (Espírito } \\
\text { Santo, Rio de Janeiro) }\end{array}$ \\
\hline Acnistus arborescens (L.) Schltdl. & $\begin{array}{l}\text { Nordeste (Alagoas, Bahia, Ceará, Paraíba, Pernambuco, Rio Grande do Norte, Sergipe), Sudeste } \\
\text { (Espírito Santo, Minas Gerais, Rio de Janeiro, São Paulo) e Sul (Paraná, Rio Grande do Sul, Santa } \\
\text { Catarina) }\end{array}$ \\
\hline Styrax acuminatus Pohl & Sudeste (Rio de Janeiro, São Paulo) e Sul (Paraná, Rio Grande do Sul, Santa Catarina) \\
\hline Citharexylum myrianthum Cham. & \begin{tabular}{l} 
Nordeste (Alagoas, Bahia, Ceará, Maranhão, Paraíba, Pernambuco, Piauí, Rio Grande do Norte, \\
Gergipe), Sudeste (Espírito Santo, Minas Gerais, Rio de Janeiro, São Paulo) e Sul (Paraná, Rio \\
\hline
\end{tabular} \\
\hline
\end{tabular}

\section{DISCUSSÃO}

Inicialmente, com a seleção de processos protocolados na SEMAS, acreditava-se que os IF's100\% tivessem sido verificados, no quesito Nome Científico das espécies florestais inventariadas, mas alguns processos continham apenas os nomes vernaculares, de acordo com os arquivos que foram acessados. Isso contradiz o estabelecido em Brasil (2009), cuja correta identificação deverá ser obrigatoriamente realizada por meios de procedimentos técnico-científicos para florestas que serão manejadas. Os IF100\% protocolados devem conter as espécies arbóreas com $\mathrm{DAP} \geq 40 \mathrm{~cm}$, considerando o diâmetro mínimo de corte estabelecido na norma (PARÁ, 2015).

O número de famílias botânicas encontrado neste estudo manteve-se próximo aos valores encontrados por Higuchi et al. (1985), que obteve uma riqueza de 51 famílias, e Rodrigues et al. (2012), que em uma comparação entre florestas secundárias e primárias, demonstrou que em florestas primárias, os números de famílias amostradas, em sua área, chegaram às 53 . Apenas o número de espécies ficou além dos quantitativos amostrados nos dois trabalhos citados. As principais famílias foram Fabaceae, Moraceae, Sapotaceae e Lauraceae, semelhante ao encontrado por Gonçalves et al. (2008).

A espécie que utiliza o nome de um pássaro (Pyrrhura frontalis (Vieil.) é um exemplo, assim como a espécie que não tem registro de ocorrência no Brasil (Sorbus domestica L.), de como o nome vernacular ganha importância na identificação dos inventários para manejo florestal, e também demonstra a falta de zelo com o nome científico, que muitas vezes é relegado. Lacerda et al. (2010) também observaram que a correlação entre nomes populares e nomes científicos é um grande problema nos inventários de planos de manejo florestal, principalmente de espécies sem valor comercial. Isso mais uma vez justifica a realização de identificações taxonômicas mais acuradas, com coleta de material botânico e depósito em herbário local, principalmente para as famílias e espécies pouco conhecidas.

Outro problema recorrente nos IF100\% é a utilização apenas de gênero e o erro na grafia ou inexistência de correlação entre gênero e epíteto específico. Vale destacar a falta de comprometimento com as identificações (nome científico) para com os táxons Psidium dentale (casca seca) e Pouteria pachycarpa Pires (goiabão). Estas espécies não constam em nenhum dos sistemas consultados e são amplamente utilizados em diversos meios, inclusive em alguns trabalhos científicos. O 'Psidium dentale' está presente em 
37\% dos inventários analisados e o Pouteria pachycarpa Pires está em $82 \%$, nos quais, em muitos está listado e autorizado para CORTE. Além disso, o táxon Pouteria pachycarpa está associado ao autor João Murça Pires, que, segundo o site Tropicos, possui nove (09) espécies do gênero Pouteria ( $P$. amapaensis, $P$. brevensis, $P$. crassiflora, P. exstaminodia, P. jariensis, P. oblanceolata, P. rodriguesiana, P. tarumanensis e P. tenuisepala) registrado em seu nome (sozinho ou com outro autor).

Verificaram-se também algumas divergências entre o Trópicos e o REFLORA, como no caso da espécie Drymaria cordata (L.) Willd. ex Roem. \& Schult., descrita por Carneiro (2019), que se encontra com esta nomenclatura no REFLORA e no Tropicos está como Drymaria cordata (L.) Willd. ex Schult., que segundo o Trópicos, justifica-se, pois, o autor Schulte estava sozinho na última publicação que referenciava a espécie. Outro caso é a da espécie Pera glabrata (Schott) Baill. que se encontra no REFLORA, mas no Tropicos possui a nomenclatura atualizada para Pera glabrata (Schott) Poepp. ex Baill.

A proposta metodológica da escolha das famílias com menor representatividade tinha como alvo as espécies de menor importância econômica, mas houve algumas famílias que se destacaram por apresentar grande frequência nos inventários analisados, dentre elas, a família Goupiaceae, com uma espécie, que é uma família monogenérica (apenas um gênero) da América do Sul, e de amplo uso, inclusive madeireiro (BIRAL, 2019; GURGEL et al., 2015). Outra família que também se destacou neste aspecto, foi a Simaroubaceae, cujo um dos representantes (Simarouba amara Aubl.) também possui amplo uso econômico (DEVECCHI et al., 2019; CRUZ et al., 2016). Ambas estavam com a nomenclatura botânica correta em alguns inventários florestais e em outros, estavam sem os nomes dos autores, mas mantinham a correta grafia para o gênero e o epíteto específico.

As outras famílias com menor representatividade apresentaram algum problema com a nomenclatura utilizada, como: falta do Autor, erro na grafia do gênero, erro na grafia do epíteto específico, erro na grafia do Autor, nomenclatura desatualizada e uso de sinônimos e/ou nomes não aceitos. Assim como espécies que não ocorrem na região norte, e outras que nem existe registro de ocorrência no Brasil. Além destas discrepâncias, as espécies que não são árvores, mas constam no IFs, reforçam o grande problema que é a correlação nome vulgar e nome científico.

Diversos autores como Botosso (2009), Lacerda et al. (2010), Martins-da-Silva (2002), Araújo (2006), Procópio et al. (2008), Ferreira et al. (2006), Vibrans (2012) e Procópio et al. (2010) são categóricos quanto aos problemas apresentados na utilização dos nomes vernaculares, mas é preciso reconhecer a importância da contribuição dos profissionais parabotânicos/parataxonomistas ou mateiros. Os próprios cientistas reconhecem a complexidade envolvida na identificação botânica através de metodologia de coleta, que requer a coleta de material fértil, geralmente em grandes alturas, munidos de diversos equipamentos que devem ser transportados dentro das florestas, seguindo todo um protocolo e que mesmo assim, por vezes, incorre na impossibilidade de identificar ao nível de espécies, às vezes chegando no máximo ao gênero. Araújo (2006) relata que em seu trabalho conseguiu chegar até a espécie (epíteto específico) em cerca de $60 \%$ dos táxons avaliados e coletados, e os outros ficaram no nível de gênero e família, se utilizando apenas de características morfológicas. 


\section{CONCLUSÕES}

A Constituição da República Federativa do Brasil de 1988 diz que as florestas são patrimônio nacional, e o inventário florestal é uma excelente ferramenta para auxiliar na conservação dos recursos naturais e manutenção das florestas Brasileiras. A metodologia e os profissionais envolvidos com a elaboração dos inventários florestais deveriam ser alvo de políticas públicas, de incentivo a formação e a construção de bancos de dados para desenvolvimento de projetos públicos de desenvolvimento de conhecimento e ações estratégicas, em parceria com as instituições de ensino e pesquisa.

Os órgãos licenciadores devem desenvolver mecanismos para assegurar a correta correlação entre nomes vernaculares e nomes científicos até que chegue um momento no desenvolvimento dos trabalhos de identificação botânica em que só o nome científico seja utilizado, caso o contrário, estaremos colocando em risco a diversidade e a preservação das espécies florestais amazônicas. Um primeiro passo seria o estreitamento das relações entre órgão licenciador e as instituições de pesquisa/ensino no desenvolvimento e/ou revisão de listas de correlação entre nomenclaturas, acrescida de outras informações que possibilitem reduzir o erro da identificação.

\section{REFERÊNCIAS}

ARAÚJO, H. J. B.. Inventário florestal a $100 \%$ em pequenas áreas sob manejo florestal madeireiro. Acta Amazônica, v.36, n.4, p.447-464, 2006.

BIRAL, L.. Goupiaceae in Flora do Brasil 2020 em construção. Jardim Botânico do Rio de Janeiro, 2019.

BRASIL. Resolução do CONAMA n. 406, de 02 de fevereiro de 2009. Estabelece parâmetros técnicos a serem adotadas na elaboração, apresentação, avaliação técnica e execução de Plano de Manejo Florestal Sustentável - PMFS com fins madeireiros, para florestas nativas e suas formas de sucessão no bioma Amazônia. Brasília: DOU, 2009.

BRASIL. Lei n. 12.651, de 25 de maio de 2012. Esta Lei estabelece normas gerais sobre a proteção da vegetação, áreas de Preservação Permanente e as áreas de Reserva Legal; a exploração florestal, o suprimento de matéria-prima florestal, o controle da origem dos produtos florestais e o controle e prevenção dos incêndios florestais, e prevê instrumentos econômicos e financeiros para o alcance de seus objetivos. Brasília: DOU, 2012.

BOTOSSO, P. C.. Identificação macroscópica de madeiras: guia prático e noções básicas para o seu reconhecimento. Colombo: Embrapa Florestas, 2009.

CARNEIRO, C. E.. Caryophyllaceae in Flora do Brasil 2020 em construção. Jardim Botânico do Rio de Janeiro, 2019.

CRUZ, E. D.; CORRÊA, J. F.. Germinação de sementes de espécies amazônicas: marupá (Simarouba amara Aubl.). Comunicado Técnico 262. Belém: EMBRAPA, 2016.

DEVECCHI, M. F.; PIRANI, J. R.; THOMAS, W. W.. Simaroubaceae in Flora do Brasil 2020 em construção. Jardim Botânico do Rio de Janeiro, 2019.
FERREIRA, G. C.; ANDRADE, A. C. S.. Diretrizes para coleta, herborização e identificação de material botânico nas parcelas permanentes em florestas naturais da Amazônia brasileira. Manaus: GT Monitoramento de Florestas, 2006.

GONÇALVES, F. G.; SANTOS, J. S.. Composição florística e estrutura de uma unidade de manejo florestal sustentável na Floresta Nacional do Tapajós, Pará. Acta Amazônica, v.38, n.2, p.229-244, 2008.

GURGEL, E. S.; GOMES, J. I.; GROPPO, M.; MARTINS-DASILVA, R. C. V.; SOUZA, A. S.; MARGALHO, L.; CARVALHO, L. T.. Conhecendo Espécie de Plantas da Amazônia: Cupiúba (Goupia glabra Aubl.- Goupiaceae). Comunicado Técnico 262. Belém: EMBRAPA, 2015.

HIGUCHI, N.; JARDIM, F. C. S.; SANTOS, J.; BARBOSA, A. P.; WOOD, T. W. W.. Inventário florestal comercial. Acta Amazônica, v.15, n.3-4, p.327-369, 1985.

JUDD, W. S.; CAMPBELL, C. S.; KELLOGG, E. A.; STEVENS, P. F.; DONOGHUE, M. J.. Sistemática Vegetal: Um Enfoque Filogenético. 3 ed. Artmed, 2009.

LACERDA, A. E. B.; NIMMO, E. R.; FARIA, B. S.; KELLERMANN B.; ALBERGONI, L.; GARRASTAZU, M. C.; OLIVEIRA, Y. M. M.; MATTOS, P. P.; ROSOT, M. A. D.. A identificação botânica no manejo florestal na Amazônia. Documento 204. Colombo: EMBRAPA, 2010.

MARTINS-DA-SILVA, R. C. V.. Coleta e identificação de espécimes botânicos. Documento 143. Belém: EMBRAPA 2002.

PARÁ. Instrução Normativa n. 005/2015, de 10 de setembro de 2015. Procedimentos técnicos para elaboração, apresentação, execução e avaliação técnica de Plano de Manejo Florestal Sustentável - PMFS nas florestas primárias, 
exploradas ou não, e suas formas de sucessão, no Estado do Pará, Secretaria de Meio Ambiente e Sustentabilidade do Pará. Belém: SEMAS, 2019.

PROCÓPIO, L. C.; SECCO, R. S.. A importância da identificação botânica nos inventários florestais: o exemplo do 'tauari' (Couratari spp. e Cariniana spp.-Lecythidaceae) em duas áreas manejadas no estado do Pará. Acta Amazônica, v.38, n.1, p.31-44, 2008.

PROCÓPIO, L. C.; GAYOT, M.; SIST, P.; FERRAZ, I. D. K.. As espécies de tauari (Lecythidaceae) em florestas de terra firme da Amazônia: padrões de distribuição geográfica, abundâncias e implicações para a conservação. Acta Bot. Bras., Feira de Santana, v.24, n.4, 2010.

QUEIROZ, W. T.. Amostragem em inventário florestal. Belém: UFRA, 2012.
RODRIGUES, S. T.; SCHWARTZ, G.; ALMEIDA, S. S.

Diversidade, síndromes de dispersão e formas de vida vegetal em diferentes estágios sucessionais de florestas secundárias em Tomé-açu, Pará, Brasil. Amazônia: Ci. \& Desenv., Belém, v.7, n.14, 2012.

SILVA, J. N. M.; CARVALHO, J. O. P.; LOPES, J. C. A.. Inventário florestal de uma área experimental na floresta nacional do tapajós. Boletim de Pesquisa Florestal, Colombo, n.10/11, p.38-110, 1985.

SOUZA, A. L.; SOAREAS, C. P. B.. Florestas Nativas: estrutura, dinâmica e manejo. Viçosa: UFV, 2013.

VIBRANS A. C.; GASPER, A. L.; MÜLLER, J. V.. Para que inventariar florestas? Reflexões sobre a finalidade do inventário florístico florestal de Santa Catarina. Revista de estudos ambientais, v.14, n.1, p.6-13, 2012. 